\title{
Impact of Olaparib for Maintenance Monotherapy on Survival in Breast and Ovarian Cancer: A Systematic Review and Pooled Analysis of Published Trials
}

\author{
Jun Dong*, Tian Zhang", Bixiu Wen" \\ Department of Radiation Oncology, The First Affiliated Hospital of Sun Yat-sen University, Guangzhou, China \\ Email:"wenbix@mail.sysu.edu.cn
}

Received 27 August 2015; accepted 8 November 2015; published 11 November 2015

Copyright (C) 2015 by authors and Scientific Research Publishing Inc. This work is licensed under the Creative Commons Attribution International License (CC BY). http://creativecommons.org/licenses/by/4.0/

(c) $)$ (i) 0 pen Access

\begin{abstract}
Purpose: To assess the efficacy and safety of Olaparib, a PARP inhibitor on progression-free survival (PFS), objective response rate (ORR) and overall survival (OS) in patients with breast and ovarian cancer. Methods: Research data from clinical trials through PubMed, Science Citation Index, Elsevier Science Direct and Cochrane Library of all published studies exploring the PFS, ORR or OS of Olaparib for maintenance monotherapy on survival in breast and ovarian cancer were analysed. Pooled estimates of the ORR, weighted medians of PFS and OS from all Olaparib were calculated. Assessment of quality and level of evidence was assigned by Cochrane guidelines and guidelines of Oxford Centre for Evidence-Based Medicine. Results: Data of 893 patients $(731$ olaparib; 162 control) from 6 trials, 2 randomised controlled trials and 4 non-randomised trials, were included. The overall median weighted PFS and OS in patients treated with Olaparib were 5.9 and 19.1 months, respectively. The pooled ORR was $25 \%$. Olaparib showed a greater effect on PFS in patients with both wild-type BRCA and BRCA mutant gene. The most common toxicity were nausea and vomiting. Conclusions: Olaparib as maintenance monotherapy for breast and ovarian cancer is associated with promising outcomes including increased response rate and improved PFS. Its potential in clinical application is needed for further investigation in phase III trials.
\end{abstract}

\section{Keywords}

Olaparib, PARP Inhibitor, Breast Cancer, Ovarian Cancer, Pooled Analysis

\footnotetext{
*Contributing equally to the work.

${ }^{*}$ Corresponding author.
}

How to cite this paper: Dong, J., Zhang, T. and Wen, B.X. (2015) Impact of Olaparib for Maintenance Monotherapy on Survival in Breast and Ovarian Cancer: A Systematic Review and Pooled Analysis of Published Trials. International Journal of Medical Physics, Clinical Engineering and Radiation Oncology, 4, 338-347. http://dx.doi.org/10.4236/ijmpcero.2015.44040 


\section{Introduction}

Breast and ovarian cancer is the leading cause of cancer-associated mortality among women. Approximately 90\% hereditary breast-ovarian cancer (HBOC) syndrome is associated with BRCA1 and BRCA2 gene mutations [1] which have been shown high-penetrance genes for a woman's risk of breast and ovarian cancer by genetic studies [2]. Research data has demonstrated that BRCAs are essential members of homologous recombination (HR) in DNA double-strand breakage (DSB) repair with a "guardian angel" function in maintaining genomic integrity and cell survival [3].

Given the fact that patient with BRCAs gene mutation is predisposing to breast-ovarian cancer, it makes an important priority to develop potential agents to target it and/or other downstream signal pathway involved in DSB repair to exert synthetic lethality. Enzyme poly (adenosine diphosphate [ADP]-ribose) polymerases (PARP) is implicated in single strand break repair that cells with BRCA-deficiency rely on PARP-dependent base excision repair (BER). PARP inhibitors (PARPi) represent a new directions for combination therapies based on the notion of synthetic lethality on HR and BER [4]. Preclinical study has demonstrated that PARPi significantly prolonged survival in BRCA-mutant mice, but did not affect survival in wild-type BRCA ones [5]. Olaparib, a potent PARP inhibitor, exhibits promise in clinical treatment of breast-ovarian cancer and cancers with defect in DNA repair pathways [6].

Accumulating evidences indicate that Olaparib holds the promise in tolerability and efficacy even in a longlasting maintenance setting [7]. The preliminary data from phase I clinical trials have shown that the clinical benefit could be achieved with the maximum tolerated dose (MTD) 400 mg twice daily [8]-[10]. Furthermore, PARP inhibition correlates with platinum sensitivity in addition to showing a benefit in platinum-resistant patients. Safety is an important issue required to focus on. Monotherapy is universally well tolerated at dosage up to the MTD of $400 \mathrm{mg}$ bid. The most common adverse effects related to Olaparib are fatigue and gastrointestinal symptoms such as nausea and vomiting [11]. Ledermann J. et al. have reported in the randomized double-blind study that maintenance treatment with Olaparib significantly extended progression-free survival (PFS, hazard ratio 0.35 with $95 \%$ CI of $0.25-0.49, P<0.001$ ), but not overall response rate (ORR) and overall survival (OS) among patients with platinum-sensitive, relapsed, high-grade serous ovarian cancer [12]. Whereas two other studies have shown that Olaparib of $400 \mathrm{mg}$ twice daily achieved higher ORR and PFS than 100 mg twice daily in BRCA-mutated advanced breast cancer and recurrent ovarian cancer, respectively [13] [14].

Although Olaparib is a new avenue of anticancer drug in the treatment and maintenance of recurrent ovarian cancer in early clinical trials, it is far from standard therapy. Given limited and contradictory clinical effects, we perform a pooled data analysis from current phase II clinical trials to investigate the efficacy and toxicity of Olaparib with different regimens. We also evaluate the consistency of these trials and intend to explore the benefit and risk for its treatment outcome.

\section{Patients and Methods}

\subsection{Search Strategy and Selected Trials}

Trials were identified on April 11, 2015 from electronic publication databases of PubMed, Science Citation Index, Elsevier Science Direct and Cochrane Library. The search strategy used the following search terms in “(((Olaparib[Title/Abstract]) AND ovarian cancer [Title/Abstract])) OR ((Olaparib[Title/Abstract]) AND breast cancer [Title/Abstract])” and defined article type as “Clinical Trial” in PubMed; “Olaparib” were used as a keyword in searching other database. The ASCO (American Society of Clinical Oncology; www.asco.org) and ESMO (European Society for Medical Oncology; www.esmo.org) annual meeting abstracts were reviewed to identify any other trials that had presented final mature (not preliminary) data and these study would be eligible for consideration in the analysis.

Eligible studies were 1) phase II clinical trials evaluating the outcome of patients taking Olaparib alone orally as maintenance treatment in breast and ovarian cancer; 2) patients included presenting with pathologically diagnosed breast or ovarian cancer; 3) germline BRCA mutation status provided. When reports overlapped or were duplicates, we retained the data with the longest follow-up. Eligible trials were independently selected by two reviewers and disagreements were resolved by the third reviewer. We excluded the trial which combined Olaparib with other drug as maintenance therapy and pretreated with Olaparib before maintenance. Besides, phase I trials were also excluded. 


\subsection{Data Extraction}

Data from the selected trials were extracted and summarized independently by two of the authors. Any discrepancy was resolved by the adjudicating senior authors. A predesigned data extraction table was used to record registration number, first author, published year, tumor type, chemotherapy regimens, patient characteristics and BRCA mutation. The following data were extracted: number of patients in each arm, PFS, ORR and OS (overall and by patient subgroup), hazard ratios (HRs) for PFS, ORR and OS and their 95\% confidence intervals (CIs) if possible; rates of major toxic effects (i.e. nausea, vomiting, fatigue, anaemia, diarrhea, constipation and abdominal pain). If HR was not directly presented, we estimated the log HR from the survival curves by the graphics software Engauge Digitizer 4.1. To assess trial quality we record the items regarding blinding, randomization including stratification factor and allocation concealment, follow-up time if possible by arm, number of patients lost to follow-up by arm and intention-to-treat analysis.

\subsection{Statistical Analysis}

Data (events) were derived directly from eligible articles, extracted from survival curves by specific software (Engauge Digitizer 4.1 and program calculating HR developed by Jayne F. Tierney et al. [15]), or calculated by percentage in each article. Data were analyzed from all patients enrolled on an intention-to-treat basis if possible. We only analyzed the patients enrolled in summary data in trials. The main outcomes are summarized using descriptive statistics. The primary endpoint was PFS defined as the continued survival in the absence of evidence for progression of disease. The secondary endpoints were carried out on ORR - the percentage of patients achieving a complete response (CR) or a partial response (PR) defined by RECIST (Response Evaluation Criteria in Solid Tumors), and OS defined as time from randomization to death and the rate of adverse effects. For all analyses of effect sizes, the main analysis included all the eligible trials.

According to Cochrane Handbook for Systematic Review of Interventions, there is no single recommended instrument for non-randomized study, for which the methods that the Collaboration recommends for randomized trials is applied. The methodological quality was assessed by a validated scale (range, 0 to 5 ) to estimate the items that affect the intervention efficacy. It was reported by Jadad et al. [16] that the scale consisted of factors: randomization methods, stratification factors, blinding, follow-up and intention-to-treat analysis.

Levels of evidence and grades of recommendation were assigned by guidelines of Oxford Centre for Evidence-Based Medicine [17] (CEBM, version March 2009).

The pooled data estimates of proportions with corresponding 95\% CIs of ORR data were calculated and showed in forest plots. All analyses were performed using Stata statistical software 12.0 (Stata Corporation, College Station, Texas, USA).

\section{Results}

Search strategy yielded 176 articles (Shown in Figure 1), 170 of them did not fulfill inclusion criteria. Reasons for exclusion were as follows: 105 were reviews, news and preclinical studies; 47 were excluded for duplicates that one or several of them reported the same trials in different articles or conference abstract; 19 publications were phase I trials, retrospective studies and other diagnoses. Six trials including 893 cases (731 cases for Olaparib and 162 cases for control) fulfilled the predefined inclusion criteria and were enrolled in the final analysis, which is finally approved by all reviewers. There is no further study for evaluation found by extensive search the references listed for these studies and for the review articles. The detailed statistical reports were available for Kaye S.B. et al. [12] [18] [19].

\subsection{Characteristics of Eligible Studies}

The characteristics for included studies are shown in Table 1. Tutt A. et al. [14] specified advanced breast cancer; Audeh M.W. et al. [12] [13] [19] prescribed Olaparib to patients with recurrent ovarian cancer; Gelmon K.A. et al. [18] required both breast and ovarian cancer but data for breast cancer was closed for no confirmed response rate; Kaufman B. et al. [20] recruited patients with advanced cancers including ovarian, breast, pancreatic and prostate cancer and we only analyzed data of ovarian and breast cancer. Germline BRCA mutation carrier was an inclusion criteria in 4 trials [13] [14] [19] [20]; while patients with or without BRCA mutation were enrolled but the status of BRCA genes was offered in other two trials [12] [18] which presented data for 


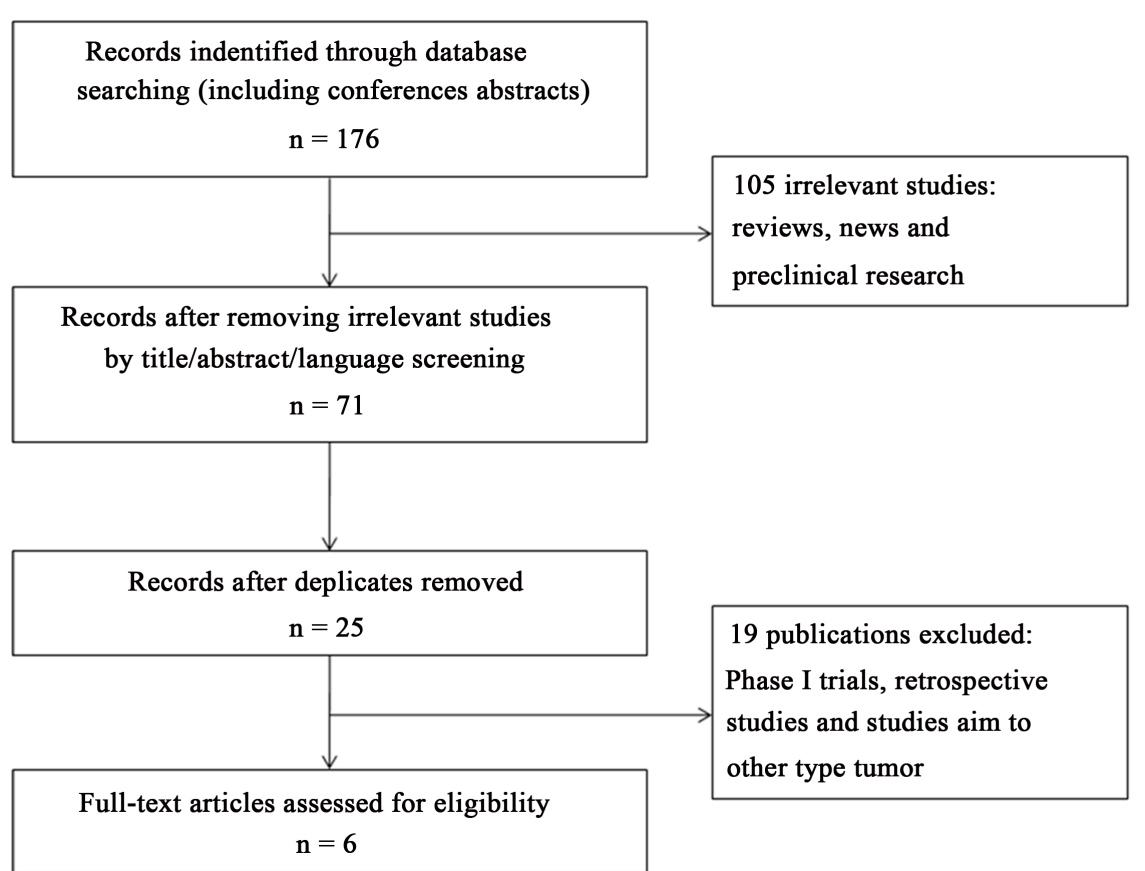

Figure 1. Selection of publications included in the pooled analysis.

Table 1. Characteristics of the six analyzed trials.

\begin{tabular}{|c|c|c|c|c|c|c|c|}
\hline Trial/Year & Design & $\begin{array}{l}\text { BRCA1/2 } \\
\text { mutation }\end{array}$ & $\begin{array}{c}\text { Primary } \\
\text { end point }\end{array}$ & Randomisation & Treatment arms & $\begin{array}{l}\text { Patients } \\
\text { in each } \\
\text { arm }\end{array}$ & Outcome \\
\hline $\begin{array}{l}\text { Andrew } \\
\text { Tutt, } \\
2010[14]\end{array}$ & $\begin{array}{c}\text { Non-randomised, } \\
\text { sequential cohort } \\
\text { design, multicentre, } \\
\text { phase II }\end{array}$ & Yes & ORR & NA & $\begin{array}{l}400 \mathrm{mg} \text { bid } \\
100 \mathrm{mg} \text { bid }\end{array}$ & $\begin{array}{l}27 \\
27\end{array}$ & $\begin{array}{c}\text { ORR was higher in } 400 \mathrm{mg} \\
\text { dosage cohort than in } 100 \\
\text { mg dosage cohort }\end{array}$ \\
\hline $\begin{array}{l}\text { M. William } \\
\text { Audeh, } \\
2010[13]\end{array}$ & $\begin{array}{l}\text { Non-randomised, } \\
\text { prospective, } \\
\text { multicentre, phase II }\end{array}$ & Yes & ORR & NA & $\begin{array}{l}400 \mathrm{mg} \text { bid } \\
100 \mathrm{mg} \text { bid }\end{array}$ & $\begin{array}{l}33 \\
24\end{array}$ & $\begin{array}{l}\text { ORRs were confirmed and } \\
\text { duration of response in the } \\
\text { two cohorts ( } 33 \% \text { vs. 13\%) }\end{array}$ \\
\hline $\begin{array}{l}\text { Karen A. } \\
\text { Gelmon, } \\
2011[18]\end{array}$ & $\begin{array}{l}\text { Non-randomised, } \\
\text { open-label, } \\
\text { multicentre, phase II }\end{array}$ & $\begin{array}{l}\text { With or } \\
\text { without }\end{array}$ & ORR & NA & $\begin{array}{l}\text { Breast cancer } \\
400 \text { mg bid } \\
\text { Ovarian cancer } \\
400 \text { mg bid }\end{array}$ & $\begin{array}{l}26 \\
65\end{array}$ & $\begin{array}{c}\text { Confirmed objective } \\
\text { responses were reported } \\
\text { in patients with ovarian } \\
\text { carcinoma ( } 29 \%) \text {, } \\
\text { particularly in } \\
\text { BRCA mutation cohort }\end{array}$ \\
\hline $\begin{array}{c}\text { Stan B. } \\
\text { Kaye. } 2012 \\
{[19]}\end{array}$ & $\begin{array}{c}\text { Randomized, } \\
\text { prospective, open-label, } \\
\text { multicenter, phase II }\end{array}$ & Yes & PFS & $1: 1: 1$ & $\begin{array}{c}200 \mathrm{mg} \text { bid } \\
400 \mathrm{mg} \text { bid } \\
\text { PLD } 50 \mathrm{mg} / \mathrm{m}^{2}\end{array}$ & $\begin{array}{l}32 \\
32 \\
33\end{array}$ & $\begin{array}{l}\text { There was no statistically } \\
\text { significant difference } \\
\text { in PFS ( } p=0.66)\end{array}$ \\
\hline $\begin{array}{c}\text { Jonathan } \\
\text { Ledermann, } \\
\mathbf{2 0 1 4} \\
{[12][23]}\end{array}$ & $\begin{array}{l}\text { Randomized, } \\
\text { double-blind, } \\
\text { phase } 2 \text { study }\end{array}$ & $\begin{array}{l}\text { With or } \\
\text { without }\end{array}$ & PFS & $1: 1$ & $\begin{array}{c}400 \text { mg bid } \\
\text { Placebo }\end{array}$ & $\begin{array}{l}136 \\
129\end{array}$ & $\begin{array}{l}\text { PFS was significantly } \\
\text { longer with olaparib than } \\
\text { with placebo }(p<0.001)\end{array}$ \\
\hline $\begin{array}{c}\text { Bella } \\
\text { Kaufman, } \\
2015[20]\end{array}$ & $\begin{array}{l}\text { Non-randomized, } \\
\text { prospective, } \\
\text { multicenter, } \\
\text { phase II }\end{array}$ & Yes & $\begin{array}{l}\text { Tumor } \\
\text { response } \\
\text { rate }\end{array}$ & NA & $\begin{array}{l}\text { Breast cancer } \\
400 \text { mg bid } \\
\text { Ovarian cancer } \\
400 \text { mg bid } \\
\text { Pancreatic cancer } \\
400 \text { mg bid } \\
\text { Prostate cancer } \\
400 \text { mg bid } \\
\text { other }\end{array}$ & $\begin{array}{l}62 \\
193 \\
23 \\
8 \\
12\end{array}$ & $\begin{array}{c}\text { Tumor response rate was } \\
31.1 \% \text { and } 12.9 \% \text { of } \\
\text { patients with ovarian } \\
\text { and breast cancers, } \\
\text { respectively }\end{array}$ \\
\hline
\end{tabular}


PFS, ORR and OS in BRCA mutation group and wild-type BRCA group. All patients had received at least one chemotherapy regimen. Chemotherapy regimens consisted of platinum-based chemotherapy, taxane therapy, anthracycline, capecitabine and targeted therapy.

Patients in all trials were continuously treated with oral Olaparib with a dosing interval of about 12 hours until disease progression or unacceptable toxicity. The regimen for Olaparib for all trials was set up with a dose of 400 mg twice per day; while Tutt A. et al. and Audeh M.W. et al. compared two dosage of Olaparib (400 mg bid and $100 \mathrm{mg}$ bid) and Kaye S.B. et al. evaluated $400 \mathrm{mg}$ bid, $200 \mathrm{mg}$ bid and pegylated liposomal doxorubicin. Summary data for the experimental arms versus the control arm were not available.

\subsection{Progression-Free Survival and Overall Survival}

Median PFS was reported in all studies (Table 2). The overall median weighted PFS in patients treated with Olaparib was 5.9 months ranging from 1.9 to 8.8 months. It was observed that low does (100 mg twice daily) leaded to a low PFS (1.9 - 3.8 months) [13] [14] and $100 \mathrm{mg}$ twice per day was the lowest PARP inhibitory dose with clinical activity. The two treatment arms may be considered as an influential factor decreasing median PFS. Median PFS in patients with BRCA mutation was 6.2 months ranging from 1.9 to 11.2 months. The overall median weighted OS was 19.1 months (range, 11.0 to 29.8 months) [12] [20]. The median overall survival was at $58 \%$ maturity in the overall population [12].

Table 2. Response to olaparib.

\begin{tabular}{|c|c|c|c|c|c|c|c|c|}
\hline \multirow[b]{2}{*}{ Trial } & \multirow{2}{*}{ Tumor type } & \multirow{2}{*}{$\begin{array}{c}\text { Treatment } \\
\text { arm }\end{array}$} & \multicolumn{2}{|c|}{ PFS } & \multicolumn{2}{|c|}{ ORR } & \multicolumn{2}{|c|}{ OS } \\
\hline & & & $\begin{array}{l}\text { Months } \\
\text { (95\% CI) }\end{array}$ & $\begin{array}{c}\text { HR } \\
(95 \% \mathrm{CI})\end{array}$ & $\begin{array}{c}\% \\
\text { (95\% CI) }\end{array}$ & $\begin{array}{c}\text { OR } \\
(95 \% \mathrm{CI})\end{array}$ & $\begin{array}{l}\text { Months } \\
\text { (95\% CI) }\end{array}$ & $\begin{array}{c}\text { HR } \\
(95 \% \mathrm{CI})\end{array}$ \\
\hline \multirow{2}{*}{$\begin{array}{l}\text { Andrew } \\
\text { Tutt [14] }\end{array}$} & \multirow{2}{*}{$\begin{array}{c}\text { Advanced breast } \\
\text { cancer }\end{array}$} & 400 mg bid & $\begin{array}{c}5.7 \\
(4.6-7.4)\end{array}$ & 0.45 & $\begin{array}{c}41 \% \\
(25-59)\end{array}$ & NA & NA & NA \\
\hline & & 100 mg bid & $\begin{array}{c}3.8 \\
(1.9-5.5)\end{array}$ & $(0.23-0.87)^{\mathrm{b}}$ & $\begin{array}{c}22 \% \\
(11-41)\end{array}$ & NA & NA & NA \\
\hline \multirow{2}{*}{$\begin{array}{l}\text { M. William } \\
\text { Audeh [13] }\end{array}$} & \multirow{2}{*}{ Ovarian cancer } & 400 mg bid & $\begin{array}{c}5.8 \\
(2.8-10.6)\end{array}$ & 0.47 & $\begin{array}{c}33 \% \\
(20-51)\end{array}$ & NA & NA & NA \\
\hline & & 100 mg bid & $\begin{array}{c}1.9 \\
(1.8-3.6)\end{array}$ & $(0.27-0.81)^{\mathrm{b}}$ & $\begin{array}{c}13 \% \\
(4-31)\end{array}$ & NA & NA & NA \\
\hline \multirow{2}{*}{$\begin{array}{c}\text { Karen } \\
\text { A. Gelmon } \\
{[18]}\end{array}$} & Breast cancer $^{\mathrm{a}}$ & 400 mg bid & $\begin{array}{l}\text { Data } \\
\text { closed }\end{array}$ & $\begin{array}{l}\text { Data } \\
\text { closed }\end{array}$ & $\begin{array}{c}\text { Data } \\
\text { closed }\end{array}$ & $\begin{array}{l}\text { Data } \\
\text { closed }\end{array}$ & $\begin{array}{c}\text { Data } \\
\text { closed }\end{array}$ & $\begin{array}{l}\text { Data } \\
\text { closed }\end{array}$ \\
\hline & Ovarian cancer & 400 mg bid & $\begin{array}{c}7.3 \\
(3.7-9.1)\end{array}$ & NA & $\begin{array}{c}29 \% \\
(19-41)\end{array}$ & NA & NA & NA \\
\hline \multirow{3}{*}{$\begin{array}{c}\text { Stan B. Kaye } \\
{[19]}\end{array}$} & \multirow{3}{*}{$\begin{array}{c}\text { Recurrent } \\
\text { ovarian } \\
\text { cancer }\end{array}$} & 200 mg bid & $\begin{array}{c}6.5 \\
(5.5-10.1)\end{array}$ & $\begin{array}{c}0.91 \\
(0.48-1.74)\end{array}$ & $25 \%$ & $\begin{array}{c}1.90 \\
(0.55-7.01)\end{array}$ & $9 / 32^{c}$ & $\begin{array}{c}0.66 \\
(0.27-1.55)\end{array}$ \\
\hline & & 400 mg bid & $\begin{array}{c}8.8 \\
(5.4-9.2)\end{array}$ & $\begin{array}{c}0.86 \\
(0.45-1.62)\end{array}$ & $31 \%$ & $\begin{array}{c}2.69 \\
(0.81-9.76)\end{array}$ & $11 / 32^{\mathrm{c}}$ & $\begin{array}{c}1.01 \\
(0.44-2.27)\end{array}$ \\
\hline & & $\begin{array}{c}\text { PLD } \\
50 \mathrm{mg} / \mathrm{m}^{2}\end{array}$ & $\begin{array}{c}7.1 \\
(3.7-10.7)\end{array}$ & & $18 \%$ & $\begin{array}{c}2.27 \\
(1.13-4.79)\end{array}$ & $13 / 33^{c}$ & \\
\hline \multirow{2}{*}{$\begin{array}{c}\text { Jonathan } \\
\text { Ledermann } \\
\text { [12] [23] }\end{array}$} & \multirow{2}{*}{$\begin{array}{c}\text { Recurrent } \\
\text { ovarian } \\
\text { cancer }\end{array}$} & 400 mg bid & $\begin{array}{c}8.4 \\
(7.4-11.5)\end{array}$ & $\begin{array}{c}0.35 \\
(0.25-0.49)\end{array}$ & $12 \%$ & $\begin{array}{c}3.36 \\
(0.75-23.72)\end{array}$ & $\begin{array}{c}29.8 \\
(27.2-35.7)^{d}\end{array}$ & $\begin{array}{c}0.88 \\
(0.64-1.21)\end{array}$ \\
\hline & & Placebo & $\begin{array}{c}4.8 \\
(4.0-5.5)\end{array}$ & & $4 \%$ & & $\begin{array}{c}27.8 \\
(24.4-34.0)\end{array}$ & \\
\hline \multirow{2}{*}{$\begin{array}{c}\text { Bella } \\
\text { Kaufman } \\
{[20]}\end{array}$} & $\begin{array}{c}\text { Advanced } \\
\text { breast cancer }\end{array}$ & 400 mg bid & 3.7 & NA & $\begin{array}{c}12.9 \% \\
(5.7-23.9)\end{array}$ & NA & 11.0 & NA \\
\hline & $\begin{array}{c}\text { Advanced } \\
\text { ovarian cancer }\end{array}$ & 400 mg bid & 7.0 & NA & $\begin{array}{c}31.1 \% \\
(24.6-38.1)\end{array}$ & NA & 16.6 & NA \\
\hline
\end{tabular}

HR: hazard ratio; OR: odds ratio; NA: not available. ${ }^{a}$ Data was closed for no comfirmed objective response; ${ }^{b}$ HR data is exacted by Engauge Digitizer 4.1 and calculated by the file according to Jayne F. Tierney et al. ${ }^{\circ}$ These data is the No. of deaths/No. of entered in each group for no median overall survival and overall survival curve in the article; ${ }^{\mathrm{d}}$ The median overall survival was at $58 \%$ maturity in the overall population. 


\subsection{Objective Response Rate}

Response assessment was done by Response Evaluation in Solid Tumors (RECIST) in all trials. The ORR was $25 \%$ (range, $12.9 \%$ to $41 \%$; P value for heterogeneity was $<0.0001$; $\mathrm{I}^{2}=77.4 \%$, random-effect model; Figure 2). At Ledermann J. et al. study entry, only $40 \%$ of the overall population had measurable disease and could be assessed for an objective response [12].

\subsection{Toxicity}

The adverse effects were reported in all trials. The common toxicities included fatigue, nausea, vomiting, diarrhea, constipation, abdominal pain, dyspepsia and anaemia (Table 3). Among these nausea (range from 36\% to $78 \%$ ) and vomiting (range from $30 \%$ to $70 \%$ ) were the most common toxicity.

\begin{tabular}{l|l} 
Study \\
ID
\end{tabular}

Figure 2. Pooled analysis for response rate. CI indicates confidence interval.

Table 3. Toxicity analysis.

\begin{tabular}{|c|c|c|c|c|c|c|c|c|c|}
\hline \multirow[b]{2}{*}{ Trials } & \multirow[b]{2}{*}{ Arm } & \multicolumn{8}{|c|}{ Events \% (No. of events/No. entered) } \\
\hline & & Nausea & Fatigue & Vomiting & Anaemia & Diarrhoea & Constipation & $\begin{array}{c}\text { Abdominal } \\
\text { pain }\end{array}$ & Dyspepsia \\
\hline \multirow{2}{*}{$\begin{array}{l}\text { Andrew } \\
\text { Tutt [14] }\end{array}$} & 400 mg bid & $\begin{array}{c}56 \% \\
(15 / 27)\end{array}$ & $\begin{array}{c}56 \% \\
(15 / 27)\end{array}$ & $\begin{array}{c}22 \% \\
(6 / 27)\end{array}$ & $\begin{array}{c}15 \% \\
(4 / 27)\end{array}$ & $\begin{array}{c}11 \% \\
(3 / 27)\end{array}$ & $\begin{array}{c}7 \% \\
(2 / 27)\end{array}$ & $7 \%(2 / 27)$ & $\begin{array}{c}7 \% \\
(2 / 27)\end{array}$ \\
\hline & 100 mg bid & $\begin{array}{c}41 \% \\
(11 / 27)\end{array}$ & $\begin{array}{c}30 \% \\
(8 / 27)\end{array}$ & $\begin{array}{c}15 \% \\
(4 / 27)\end{array}$ & $\begin{array}{c}15 \% \\
(4 / 27)\end{array}$ & $\begin{array}{c}7 \% \\
(2 / 27)\end{array}$ & $\begin{array}{c}15 \% \\
(4 / 27)\end{array}$ & $11 \%(3 / 27)$ & $\begin{array}{c}4 \% \\
(1 / 27)\end{array}$ \\
\hline \multirow{2}{*}{$\begin{array}{l}\text { M. William } \\
\text { Audeh [13] }\end{array}$} & 400 mg bid & $\begin{array}{c}48 \% \\
(16 / 33)\end{array}$ & $\begin{array}{c}33 \% \\
(11 / 33)\end{array}$ & $\begin{array}{c}12 \% \\
(4 / 33)\end{array}$ & $\begin{array}{c}18 \% \\
(6 / 33)\end{array}$ & $\begin{array}{c}15 \% \\
(5 / 33)\end{array}$ & $\begin{array}{c}3 \% \\
(1 / 33)\end{array}$ & $9 \%(3 / 33)$ & $\begin{array}{c}6 \% \\
(2 / 33)\end{array}$ \\
\hline & 100 mg bid & $\begin{array}{c}37 \% \\
(9 / 24)\end{array}$ & $\begin{array}{c}37 \% \\
(9 / 24)\end{array}$ & $\begin{array}{c}0 \% \\
(0 / 24)\end{array}$ & $\begin{array}{c}17 \% \\
(4 / 24)\end{array}$ & $\begin{array}{c}12 \% \\
(3 / 24)\end{array}$ & $\begin{array}{c}8 \% \\
(2 / 24)\end{array}$ & $0 \%(0 / 24)$ & $\begin{array}{c}12 \% \\
(3 / 24)\end{array}$ \\
\hline $\begin{array}{c}\text { Karen A. } \\
\text { Gelmon [18] }\end{array}$ & $\begin{array}{l}\text { Ovarian cancer } \\
400 \text { mg bid }\end{array}$ & $\begin{array}{c}66 \% \\
(42 / 64)\end{array}$ & $\begin{array}{c}70 \% \\
(45 / 64)\end{array}$ & $\begin{array}{c}39 \% \\
(25 / 64)\end{array}$ & NA & $\begin{array}{c}23 \% \\
(15 / 64)\end{array}$ & NA & $20 \%(13 / 64)$ & $\begin{array}{c}23 \% \\
(15 / 64)\end{array}$ \\
\hline \multirow{3}{*}{$\begin{array}{c}\text { Stan B. Kaye } \\
{[19]}\end{array}$} & 200 mg bid & $\begin{array}{c}59 \% \\
(19 / 32)\end{array}$ & $\begin{array}{c}41 \% \\
(13 / 32)\end{array}$ & $\begin{array}{c}34 \% \\
(11 / 32)\end{array}$ & $\begin{array}{c}12 \% \\
(4 / 32)\end{array}$ & $\begin{array}{c}19 \% \\
(6 / 32)\end{array}$ & $\begin{array}{c}28 \% \\
(9 / 32)\end{array}$ & $37 \%(12 / 32)$ & NA \\
\hline & 400 mg bid & $\begin{array}{c}78 \% \\
(25 / 32)\end{array}$ & $\begin{array}{c}66 \% \\
(21 / 32)\end{array}$ & $\begin{array}{c}50 \% \\
(16 / 32)\end{array}$ & $\begin{array}{c}31 \% \\
(10 / 32)\end{array}$ & $\begin{array}{c}37 \% \\
(12 / 32)\end{array}$ & $\begin{array}{l}16 \% \\
(5 / 32)\end{array}$ & $25 \%(8 / 32)$ & NA \\
\hline & $\mathrm{PLD} 50 \mathrm{mg} / \mathrm{m}^{2}$ & $\begin{array}{c}54 \% \\
(18 / 33)\end{array}$ & $\begin{array}{c}45 \% \\
(15 / 33)\end{array}$ & $30 \%(10 / 33)$ & $3 \%(1 / 33)$ & $\begin{array}{c}30 \% \\
(10 / 33)\end{array}$ & $\begin{array}{c}36 \% \\
(12 / 33)\end{array}$ & $\begin{array}{c}36 \% \\
(12 / 33)\end{array}$ & NA \\
\hline \multirow{2}{*}{$\begin{array}{c}\text { Jonathan } \\
\text { Ledermann } \\
\text { [12] [23] }\end{array}$} & 400 mg bid & $\begin{array}{c}71 \% \\
(96 / 136)\end{array}$ & $\begin{array}{c}52 \% \\
(71 / 136)\end{array}$ & $\begin{array}{c}34 \% \\
(46 / 136)\end{array}$ & $\begin{array}{c}21 \% \\
(29 / 136)\end{array}$ & $\begin{array}{c}27 \% \\
(37 / 136)\end{array}$ & $\begin{array}{c}21 \% \\
(28 / 136)\end{array}$ & $\begin{array}{c}25 \% \\
(34 / 136)\end{array}$ & $\begin{array}{c}18 \% \\
(24 / 136)\end{array}$ \\
\hline & Placebo & $\begin{array}{c}36 \% \\
(46 / 129)\end{array}$ & $\begin{array}{c}39 \% \\
(50 / 129)\end{array}$ & $\begin{array}{c}14 \% \\
(18 / 129)\end{array}$ & 5\% (7/129) & $\begin{array}{c}24 \% \\
(31 / 129)\end{array}$ & $\begin{array}{c}11 \% \\
(14 / 129)\end{array}$ & $\begin{array}{c}26 \% \\
(34 / 129)\end{array}$ & $\begin{array}{c}8 \% \\
(11 / 129)\end{array}$ \\
\hline \multirow{2}{*}{$\begin{array}{c}\text { Bella } \\
\text { Kaufman } \\
{[20]}\end{array}$} & $\begin{array}{c}\text { Breast cancer } \\
400 \text { mg bid }\end{array}$ & $\begin{array}{c}53 \% \\
(33 / 62)\end{array}$ & $\begin{array}{c}48 \% \\
(30 / 62)\end{array}$ & $34 \%(21 / 62)$ & $\begin{array}{c}26 \% \\
(16 / 62)\end{array}$ & $\begin{array}{c}18 \% \\
(11 / 62)\end{array}$ & NA & $\begin{array}{c}8 \% \\
(5 / 62)\end{array}$ & $\begin{array}{c}14 \% \\
(9 / 62)\end{array}$ \\
\hline & $\begin{array}{l}\text { Ovarian cancer } \\
400 \text { mg bid }\end{array}$ & $\begin{array}{c}62 \% \\
(119 / 193)\end{array}$ & $\begin{array}{c}60 \% \\
(116 / 193)\end{array}$ & $\begin{array}{c}39 \% \\
(75 / 193)\end{array}$ & $\begin{array}{c}32 \% \\
(62 / 193)\end{array}$ & $\begin{array}{c}29 \% \\
(56 / 193)\end{array}$ & NA & $\begin{array}{c}30 \% \\
(58 / 193)\end{array}$ & $\begin{array}{c}20 \% \\
(38 / 193)\end{array}$ \\
\hline
\end{tabular}




\subsection{Assessment of Quality of Studies and Level of Evidence}

The review of the articles revealed 2 randomized controlled trials and 4 non-randomized trials (Table 4). Only Ledermann J. et al. was double-blind. However, all the primary endpoints, PFS or ORR were objective evaluations, so the absence of blinding was not a problem. Five trials were intent to treat analysis except Gelmon K.A. et al. The proportion of randomly assigned patients excluded from the analysis overall was $0.2 \%$ (Table 4 ).

Among these studies 2 trials (33.3\%) [18] [20] are considered as single-arm trials for the reason that both of them set up only one does ( $400 \mathrm{mg}$ twice daily) for different type of tumor. They achieved level 4 evidence according to guidelines of Oxford Centre for Evidence-Based Medicine owing to failed to clearly define comparison groups.

\section{Discussions}

To our best knowledge it is the first systematic review to evaluate the efficacy and safety of Olaparib for maintenance monotherapy in breast and ovarian cancer. This pooled data analysis included 6 trials that involved in 893 patients with advanced breast cancer or recurrent breast and ovarian cancer treated with Olaparib or other anticancer agents as maintenance therapy and its results indicates that Olaparib shows promise as an effective and tolerable treatment. In addition, there is the advantage of gaining pooled data analysis to objective describe the PFS, ORR and OS, although there is a lack of homogeneity among the trials in many respects.

We focused on the monotherapy of Olaparib for the reason that combination therapy for advanced breast and relapsed ovarian may achieve higher PFS and response rates but considerable higher incidence of adverse events should not be ignored [21]. However, a study which compared efficacy between Olaparib alone and combination of Olaparib and Cediranib in recurrent platinum-sensitive ovarian cancer has shown that PFS improved significantly but grade 3 and 4 adverse events were more common in combination therapy group [22].

As a single agent, Olaparib has been shown to induce a response and beneficial effect in both BRCA-mutated and wild-type BRCA patients [23]. Ledermann J. et al. reported the largest trial comparing efficacy between Olaparib and placebo. The patients with platinum-sensitive relapsed serous ovarian cancer were enrolled. "Platinum-sensitive" relapse means that patients relapse more than six months after completion of first-line chemotherapy and further platinum-based treatment is usually given when relapsing [24] [25]. However, recent research showed that non-platinum combination therapy was considered as a new choice. In a randomized clinical trial, pegylated liposomal doxorubicin alone or in combination with Trabectedin, an anti-tumor drug resulting in DNA backbone cleavage and cell apoptosis, was given and the risk of death significantly decreased [26]. In Ledermann J. et al, Olaparib also showed an improvement in increasing PFS (HR for progression, 0.35; 95\% CI, 0.25 to $0.47 ; P<0.001$ ), but it is not able to show a difference in OS for the data was $58 \%$ maturity, meaning that $38 \%$ of the patients had died at an interim analysis in 2012 when 101 death were recorded [12] [23]. However, both of them indicated an intriguing regimen that may be a feasible alternative to platinum-based treatment.

Table 4. Quality of the four analyzed trials.

\begin{tabular}{|c|c|c|c|c|c|c|}
\hline Trials & $\begin{array}{l}\text { Randomisation } \\
\text { methods }\end{array}$ & Double-blind & Follow-up & Intent to treat & $\begin{array}{l}\text { Quality } \\
\text { (score) }^{\mathrm{a}}\end{array}$ & $\begin{array}{c}\text { Level of } \\
\text { evidence }^{\mathrm{b}}\end{array}$ \\
\hline Andrew Tutt [14] & non-randomised & No & No patients was lost. & Yes & 1 & 1 \\
\hline M. William Audeh [13] & non-randomised & No & No patients was lost. & Yes & 1 & 1 \\
\hline Karen A. Gelmon [18] & non-randomised & No & No patients was lost. & $\begin{array}{l}2 \text { of } 65 \text { excluded } \\
\text { from analysis }\end{array}$ & 1 & 4 \\
\hline Stan B. Kaye [19] & $\begin{array}{c}\text { Global } \\
\text { Randomization system }\end{array}$ & No & NA & Yes & 4 & 1 \\
\hline $\begin{array}{l}\text { Jonathan } \\
\text { Ledermann } \\
\text { [12] [23] }\end{array}$ & NA & Yes & $\begin{array}{l}\text { Rate of loss to follow-up } \\
2.2 \% \text { in olaparib group } \\
3.9 \% \text { in placebo group }\end{array}$ & Yes & 5 & 1 \\
\hline Bella Kaufman [20] & non-randomised & No & NA & Yes & 0 & 4 \\
\hline
\end{tabular}

NA: not available. ${ }^{\mathrm{a}}$ Assessed by Jadad score; ${ }^{\mathrm{b}}$ Assessed by Oxford Centre for Evidence-based Medicine-Levels of Evidence (March 2009), The CEBM "Levels of Evidence 1" document sets out one approach to systematising this process for different question types. 
PARPi is one of the most exciting advances in the management of recurrent ovarian cancer and advanced breast cancer. The application of the synthetic lethal effect of PARPi in ovarian cancer is based on the fact that $15 \%$ of the ovarian cancer population carry germline BRCA mutations and $30 \%$ to $40 \%$ show phenotypic "BRCAness" [21]. BRCA mutation is related to an elevated risk of contralateral and ipsilateral breast cancer recurrence [27]. As a result, four trials defined that BRCA-mutated was an inclusion (Table 1). Ledermann J. et al. carried out a subgroup analysis in which patients were stratified according to their BRCA status. The data showed that when comparing with placebo, Olaparib significantly increased the PFS in both BRCA mutation and wild-type groups (15). Unfortunately, there was no trial comparing the efficacy of Olaparib between BRCA mutation and wild-type BRCA subgroup. In general, there is a positive proof of application of Olaparib but further investigation is needed.

The primary limitation of our review is that it is composed mainly of literature-based series and most of the results are descriptive. Considering the fact that heterogeneity of these studies is hard to estimate, so that statistical analysis was not performed. Study design and primary endpoints of researches are different, and BRCA status differs in these studies. All of these are the source of heterogeneity. Besides, the treatment arms differed among different studies. All of these trials were set up with a $400 \mathrm{mg}$ twice daily group, while some were set up with low dosage or placebo group, and some were single-arm. This posed an additional source of bias to the analysis. Based on these reasons, meta-analysis and heterogeneity analysis cannot be performed.

Over the past years, increasingly potent PARPi have been developed and evaluated such as Iniparib and Niraparib [28]. In a phase III RCT, Iniparib was given to metastatic triple negative breast cancer [29]. This suggests that PARPi are very likely to play a crucial role in the treatment in patients carrying BRCA-mutant gene. Moreover, two phase III clinical trials of Olaparib in ovarian cancer patients with a BRCA1/2 mutation are ongoing [30] and EMA has grant it for the treatment of patients with platinum-sensitive ovarian cancer and BRCA mutations.

\section{Conclusion}

In summary, the systematic review and pooled data analysis demonstrates that patients with advanced breast cancer and recurrent ovarian cancer are now surviving longer than before, partly due to the judicious use of targeted agent particularly PARPi. Olaparib confirms a response rate and may improve PFS in patients with BRCA mutant breast and ovarian cancer. It implies a brighten and promising future of BRCA-mutant cancer treatment and long term follow-up and randomized trials are urgently needed.

\section{Fund}

This study was funded by the National Natural Science Foundation of China (grant number 81172209).

\section{Conflict of Interest}

None.

\section{References}

[1] Lynch, H.T., Casey, M.J., Snyder, C.L., et al. (2009) Hereditary Ovarian Carcinoma: Heterogeneity, Molecular Genetics, Pathology, and Management. Molecular Oncology, 3, 97-137. http://dx.doi.org/10.1016/j.molonc.2009.02.004

[2] Narod, S.A. (2011) Genetic Variants Associated with Breast-Cancer Risk. The Lancet Oncology, 12, 415-416. http://dx.doi.org/10.1016/S1470-2045(11)70084-5

[3] Lord, C.J. and Ashworth, A. (2013) Mechanisms of Resistance to Therapies Targeting BRCA-Mutant Cancers. Nature Medicine, 19, 1381-1388. http://dx.doi.org/10.1038/nm.3369

[4] Lee, J.M., Ledermann, J.A. and Kohn, E.C. (2014) PARP Inhibitors for BRCA1/2 Mutation-Associated and BRCALike Malignancies. Annals of Oncology, 25, 32-40. http://dx.doi.org/10.1093/annonc/mdt384

[5] Karginova, O., Siegel, M.B., Van Swearingen, A.E., et al. (2015) Efficacy of Carboplatin Alone and in Combination with ABT888 in Intracranial Murine Models of BRCA-Mutated and BRCA-Wild-Type Triple-Negative Breast Cancer. Molecular Cancer Therapeutics, 14, 920-930. http://dx.doi.org/10.1158/1535-7163.MCT-14-0474

[6] Rouleau, M., Patel, A., Hendzel, M.J., Kaufmann, S.H. and Poirier, G.G. (2010) PARP Inhibition: PARP1 and Beyond. Nature Reviews Cancer, 10, 293-301. http://dx.doi.org/10.1038/nrc2812 
[7] Shaw, H.M. and Hall, M. (2013) Emerging Treatment Options for Recurrent Ovarian Cancer: The Potential Role of Olaparib. OncoTargets and Therapy, 6, 1197-1206.

[8] Yamamoto, N., Nokihara, H., Yamada, Y., et al. (2012) A Phase I, Dose-Finding and Pharmacokinetic Study of Olaparib (AZD2281) in Japanese Patients with Advanced Solid Tumors. Cancer Science, 103, 504-509. http://dx.doi.org/10.1111/j.1349-7006.2011.02179.x

[9] Fong, P.C., Yap, T.A., Boss, D.S., et al. (2010) Poly(ADP)-Ribose Polymerase Inhibition: Frequent Durable Responses in BRCA Carrier Ovarian Cancer Correlating with Platinum-Free Interval. Journal of Clinical Oncology, 28, 2512-2519. http://dx.doi.org/10.1200/JCO.2009.26.9589

[10] Fong, P.C., Boss, D.S., Yap, T.A., et al. (2009) Inhibition of Poly(ADP-Ribose) Polymerase in Tumors from BRCA Mutation Carriers. The New England Journal of Medicine, 361, 123-134. http://dx.doi.org/10.1056/NEJMoa0900212

[11] Marchetti, C., Imperiale, L., Gasparri, M.L., Palaia, I., Pignata, S., Boni, T., et al. (2012) Olaparib, PARP1 Inhibitor in Ovarian Cancer. Expert Opinion on Investigational Drugs, 21, 1575-1584. http://dx.doi.org/10.1517/13543784.2012.707189

[12] Ledermann, J., Harter, P., Gourley, C., Friedlander, M., Vergote, I., Rustin, G., et al. (2012) Olaparib Maintenance Therapy in Platinum-Sensitive Relapsed Ovarian Cancer. New England Journal of Medicine, 366, 1382-1392. http://dx.doi.org/10.1056/NEJMoa1105535

[13] Audeh, M.W., Carmichael, J., Penson, R.T., Friedlander, M., Powell, B., Bell-McGuinn, K.M., et al. (2010) Oral Poly(ADP-ribose) Polymerase Inhibitor Olaparib in Patients with BRCA1 or BRCA2 Mutations and Recurrent Ovarian Cancer: A Proof-of-Concept Trial. The Lancet, 376, 245-251. http://dx.doi.org/10.1016/S0140-6736(10)60893-8

[14] Tutt, A., Robson, M., Garber, J.E., Domchek, S.M., Audeh, M.W., Weitzel, J.N., et al. (2010) Oral Poly(ADP-ribose) Polymerase Inhibitor Olaparib in Patients with BRCA1 or BRCA2 Mutations and Advanced Breast Cancer: A Proof-ofConcept Trial. The Lancet, 376, 235-244. http://dx.doi.org/10.1016/S0140-6736(10)60892-6

[15] Mahaney, B.L., Meek, K. and Lees-Miller, S.P. (2009) Repair of Ionizing Radiation-Induced DNA Double-Strand Breaks by Non-Homologous End-Joining. Biochemical Journal, 417, 639-650. http://dx.doi.org/10.1042/BJ20080413

[16] Jadad, A.R., Moore, R.A., Carroll, D., Jenkinson, C., Reynolds, D.J.M., Gavaghan, D.J. and McQuay, H.J. (1996) Assessing the Quality of Reports of Randomized Clinical Trials: Is Blinding Necessary? Controlled Clinical Trials, 17, 1-12. http://dx.doi.org/10.1016/0197-2456(95)00134-4

[17] Phillips, B., Ball, C., Sackett, D., Badenoch, D., Straus, S., Haynes, B. and Dawes, M. (2009) Oxford Centre for Evidence-Based Medicine-Levels of Evidence (March 2009). http://www.cebm.net

[18] Gelmon, K.A., Tischkowitz, M., Mackay, H., Swenerton, K., Robidoux, A., Tonkin, K., et al. (2011) Olaparib in Patients with Recurrent High-Grade Serous or Poorly Differentiated Ovarian Carcinoma or Triple-Negative Breast Cancer: A Phase 2, Multicentre, Open-Label, Non-Randomised Study. The Lancet Oncology, 12, 852-861. http://dx.doi.org/10.1016/S1470-2045(11)70214-5

[19] Kaye, S.B., Lubinski, J., Matulonis, U., Ang, J.E., Gourley, C., Karlan, B.Y., et al. (2012) Phase II, Open-Label, Randomized, Multicenter Study Comparing the Efficacy and Safety of Olaparib, a Poly(ADP-ribose) Polymerase Inhibitor, and Pegylated Liposomal Doxorubicin in Patients with BRCA1 or BRCA2 Mutations and Recurrent Ovarian Cancer. Journal of Clinical Oncology, 30, 372-379. http://dx.doi.org/10.1200/JCO.2011.36.9215

[20] Kaufman, B., Shapira-Frommer, R., Schmutzler, R.K., Audeh, M.W., Friedlander, M., Balmaña, J., et al. (2015) Olaparib Monotherapy in Patients with Advanced Cancer and a Germline BRCA1/2 Mutation. Journal of Clinical Oncology, 33, 244-250. http://dx.doi.org/10.1200/JCO.2014.56.2728

[21] Hall, M. and Rustin, G. (2011) Recurrent Ovarian Cancer: When and How to Treat. Current Oncology Reports, 13, 459-471. http://dx.doi.org/10.1007/s11912-011-0199-3

[22] Liu, J.F., Barry, W.T., Birrer, M., Lee, J.-M., Buckanovich, R.J., Fleming, G.F., et al. (2014) Combination Cediranib and Olaparib versus Olaparib Alone for Women with Recurrent Platinum-Sensitive Ovarian Cancer: A Randomised Phase 2 Study. The Lancet Oncology, 15, 1207-1214. http://dx.doi.org/10.1016/S1470-2045(14)70391-2

[23] Ledermann, J., Harter, P., Gourley, C., Friedlander, M., Vergote, I., Rustin, G., et al. (2014) Olaparib Maintenance Therapy in Patients with Platinum-Sensitive Relapsed Serous Ovarian Cancer: A Preplanned Retrospective Analysis of Outcomes by BRCA Status in a Randomised Phase 2 Trial. The Lancet Oncology, 15, 852-861. http://dx.doi.org/10.1016/S1470-2045(14)70228-1

[24] Ledermann, J.A. and Raja, F.A. (2011) Clinical Trials and Decision-Making Strategies for Optimal Treatment of Relapsed Ovarian Cancer. European Journal of Cancer, 47, S104-S115. http://dx.doi.org/10.1016/s0959-8049(11)70154-x

[25] Raja, F.A., Counsell, N., Colombo, N., Pfisterer, J., du Bois, A., Parmar, M.K., et al. (2013) Platinum versus Platinum-Combination Chemotherapy in Platinum-Sensitive Recurrent Ovarian Cancer: A Meta-Analysis Using Individual Patient Data. Annals of Oncology, 24, 3028-3034. http://dx.doi.org/10.1093/annonc/mdt406 
[26] Monk, B.J., Herzog, T.J., Kaye, S.B., Krasner, C.N., Vermorken, J.B., Muggia, F.M., et al. (2010) Trabectedin plus Pegylated Liposomal Doxorubicin in Recurrent Ovarian Cancer. Journal of Clinical Oncology, 28, 3107-3114. http://dx.doi.org/10.1200/JCO.2009.25.4037

[27] Bayraktar, S. and Gluck, S. (2012) Systemic Therapy Options in BRCA Mutation-Associated Breast Cancer. Breast Cancer Research and Treatment, 135, 355-366. http://dx.doi.org/10.1007/s10549-012-2158-6

[28] Sandhu, S.K., Schelman, W.R., Wilding, G., Moreno, V., Baird, R.D., Miranda, S., et al. (2013) The Poly(ADP-ribose) Polymerase Inhibitor Niraparib (MK4827) in BRCA Mutation Carriers and Patients with Sporadic Cancer: A Phase 1 Dose-Escalation Trial. The Lancet Oncology, 14, 882-892. http://dx.doi.org/10.1016/S1470-2045(13)70240-7

[29] O’Shaughnessy, J., Schwartzberg, L., Danso, M.A., Miller, K.D., Rugo, H.S., Neubauer, M., et al. (2014) Phase III Study of Iniparib plus Gemcitabine and Carboplatin versus Gemcitabine and Carboplatin in Patients with Metastatic Triple-Negative Breast Cancer. Journal of Clinical Oncology, 32, 3840-3847. http://dx.doi.org/10.1200/JCO.2014.55.2984

[30] Moore, K.N., DiSilvestro, P. and Lowe, E.S. (2014) SOLO1 and SOLO2 Randomized Phase III Trials of Olaparib in Patients (pts) with Ovarian Cancer and a BRCA12 Mutation (BRCAm). ASCO Annual Meeting 2014, Chicago, 30 May-3 June 2014, TPS5616 [Abstract]. 